\title{
Acquired pigmentation simulating Peutz-Jeghers syndrome: initial manifestation of diffuse uveal melanocytic proliferation
}

\author{
J Donald M Gass, Ronald J Glatzer
}

\begin{abstract}
A 74-year-old man with an occult carcinoma of the colon developed pigmentation of the mouth and penis typical of the Peutz-Jeghers syndrome as the first manifestation of bilateral diffuse uveal melanocytic proliferation. The simultaneous appearance of extraocular pigmented lesion and those in the uveal tract of both eyes of this patient provides further evidence that bilateral diffuse uveal melanocytic proliferation may be caused by activation of occult melanocytic naevus cells in response to either a hormone-producing carcinoma or to some other common oncogenic stimulus.
\end{abstract}

Bilateral diffuse uveal melanocytic proliferation (BDUMP) is a paraneoplastic disorder causing visual loss in both eyes of patients with systemic, often occult carcinomas. ${ }^{1-4}$ It is characterised by the development of (1) multiple subtle round and oval subretinal red patches that angiographically show a striking pattern of early hyperfluorescence; (2) multiple, slightly elevated, pigmented and non-pigmented, uveal melanocytic tumours, as well as evidence of diffuse thickening of the uveal tract; (3) exudative retinal detachment; and (4) rapid development of cataracts. The uveal melanocytic infiltration is composed of predominantly benign naevus cells. Metastasis of the uveal tumour has not been reported. Death caused by metastatic carcinoma usually occurs within 12 to 24 months. The cause of the ocular changes is unknown, and treatment is ineffective.

This report concerns a patient whose first sign of the BDUMP syndrome was the development of hyperpigmented patches on the mucous membranes of the mouth and penis in a pattern typical for the Peutz-Jeghers syndrome. It is the first report of simultaneous development of nonocular pigmentation in a patient with BDUMP.

\section{Case report}

In April 1988 an apparently healthy 74-year-old man developed hyperpigmented macules on his lips and penis. A dermatologist made a diagnosis of Peutz-Jeghers syndrome and recommended examination to exclude intestinal polyps. A biopsy of the lip lesions revealed labial lentigo compatible with this syndrome. Gastrointestinal investigation revealed a rectosigmoidal tumour. Biopsy showed an adenocarcinoma arising in an adenomatous polyp. In May he had a rectosigmoidal resection. There was carcinomatous involvement in two paracolonic lymph nodes. External beam irradiation and chemotherapy were instituted but were discontinued after a total dose of $3780 \mathrm{R}$ to the abdominal area and after three intravenous injections of $800 \mathrm{mg} \mathrm{5-}$ fluoro-2,4 $(1 \mathrm{H}, 3 \mathrm{H})$-pyrimidinedione because of severe gastrointestinal symptoms. The patient noted progressive loss of vision in both eyes. In September 1988 cataract extraction failed to improve his vision.

When seen here in January 1989 he had multiple bluish brown macules of the mucous membranes of lips and mouth. They were most prominent on the inferior lip and hard palate (Fig 1). Similar lesions were present on the mucous membranes and skin of the penis (Fig 2). His family history was unknown. Visual acuity in the right eye was $1 / 200$, left eye $20 / 60$. There was $1+$ episcleral injection bilaterally. Intraocular pressure was $10 \mathrm{~mm} \mathrm{Hg}$ bilaterally. Funduscopic examination revealed ciliochoroidal elevation superiorly and temporally in both eyes. There were three slightly elevated pigmented choroidal lesions of 1 to 1.5 disc diameter size in the midperiphery of both eyes (Fig 3). There was a reticular pattern of hyperpigmentation and depigmentation of the pigment epithelium that was most evident in the macula and inferior fundi. Serous detachment of the retina was present inferiorly in the right eye.

Angiography revealed a reticular pattern of non-fluorescence on a background of marked hyperfluorescence throughout the posterior fundus and midperiphery of both eyes (Figs 4, 5). An electroretinogram showed severely abnormal rod and cone function in both eyes. Serological examinations revealed antibodies reactive with the retinal protein, cancer associated retinal antigen, at a dilution of $1: 200$. $^{5}$

The patient experienced progressive loss of vision and developed three slightly raised black skin lesions in the postauricular area. In December 1989 the visual acuity in the right eye

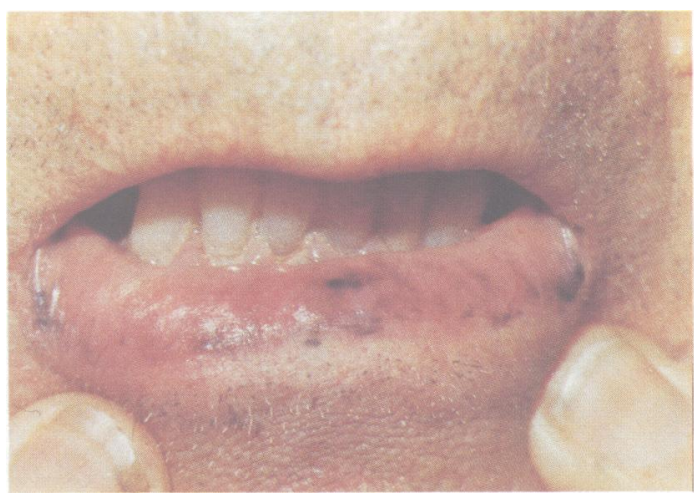

Figure 1 Pigmented macules lower lip. 


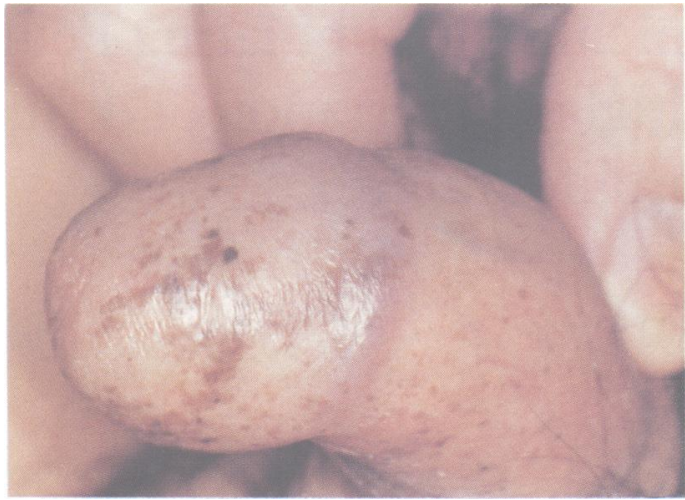

Figure 2 Pigmented macules penis.

was light perception with projection and in the left eye was hand movements only. The mucous membrane lesions were unchanged. The anterior chambers were shallow peripherally. He had a mature cataract in the right eye. The intraocular pressure was $10 \mathrm{~mm} \mathrm{Hg}$ in each eye. In the left eye there was a total bullous retinal detachment with shifting subretinal fluid. The pigmented choroidal lesions noted previously were unchanged. There were two new pigmented choroidal lesions in the perimacular area and extensive pigment mottling throughout the fundus. Echography revealed total retinal detachment and diffuse as well as multifocal areas of thickening of the choroid and ciliary body bilaterally.

Biopsy of the pigmented lesion of the lower lip revealed hyperpigmentation of the basal cell layer, melanocytic hyperplasia, irregular elongation of the rete ridges, and scattered melanocytes and melanophages in the underlying dermis (Fig 6). Electron microscopy revealed melanocytes containing mostly mature melanosomes interspersed among the keratinocytes. Adjacent keratinocytes displayed scattered round and oval mature melanosomes. The underlying stroma contained scattered macrophages filled with numerous melanosomes. The histopathological diagnosis was lentigo labialis. Biopsy of one post-auricular lesion revealed a pigmented basal cell cacinoma with some necrosis. In December 1989 the patient had drainage of the subretinal fluid, total vitreous replacement with silicone, and panretinal photocoagulation in the left eye.

When he was last examined, in February

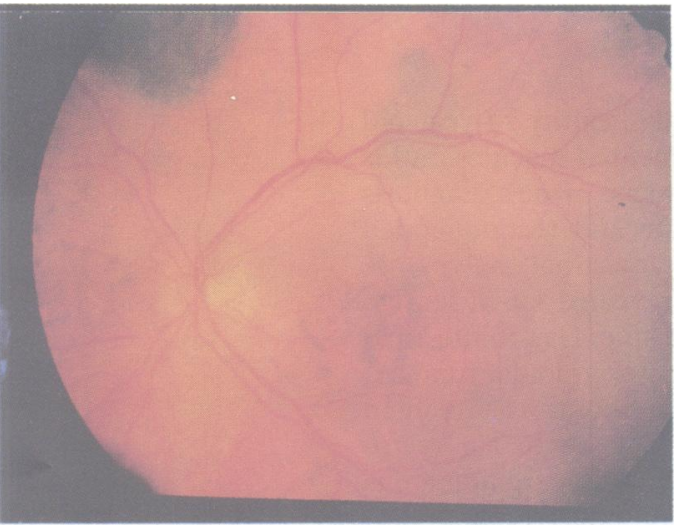

Figure 3 Left eye.

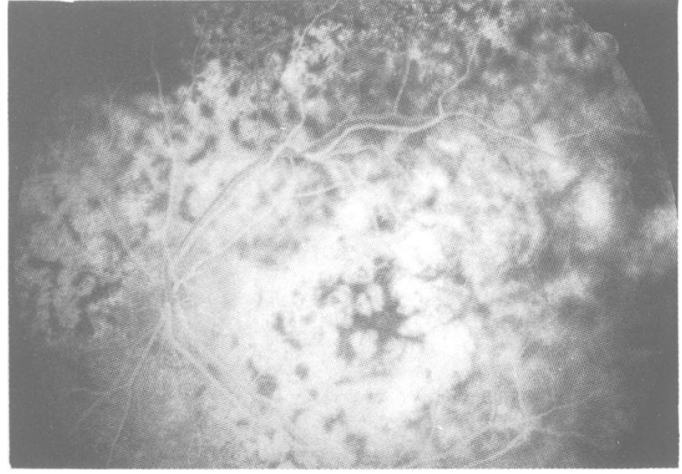

Figure 4 Macular region. Triradiate pattern of nonfluorescence caused by clumps of subretinal pigment on background of early hyperfluorescence, indicating severe pigment epithelial damage.

1990, the visual acuity in the left eye was counting fingers at $1 \mathrm{~m}$. The retina of the left eye was reattached except for a small area anterior to equator inferiorly. The pigmented mucous membrane and choroidal lesions in the left eye were unchanged. He noted no further change in his visual function or general health until June 1990, when he developed a papulonodular skin rash involving the lower trunk and legs. Biopsy revealed metastatic carcinoma to the skin.

He died in November 1990. Histopathological examination of both eyes revealed diffuse uveal tract infiltrates with apparently benign melanocytic cells in a pattern identical to that previously described. ${ }^{24}$

\section{Discussion}

Peutz-Jeghers syndrome is an autosomal dominantly inherited disorder characterised by the association of mucocutaneous pigmented lesions and gastrointestinal hamartomatous polyps involving primarily the jejunum and ileum. ${ }^{6-11}$ Multiple round and oval, 1 to $12 \mathrm{~mm}$, blue-black or dark brown macules typically are present on the lips, particularly the lower one, and are often evident in the mucous membranes of the gums, hard palate, genitalia, and perianal region. They may also be evident on the face and hands. The lids and conjunctiva may be involved. ${ }^{7811}$ The pigmented lesions are usually present in early childhood but may develop later. ${ }^{9}$ Those on the oral mucosa remain constant, whereas those elsewhere may often fade.

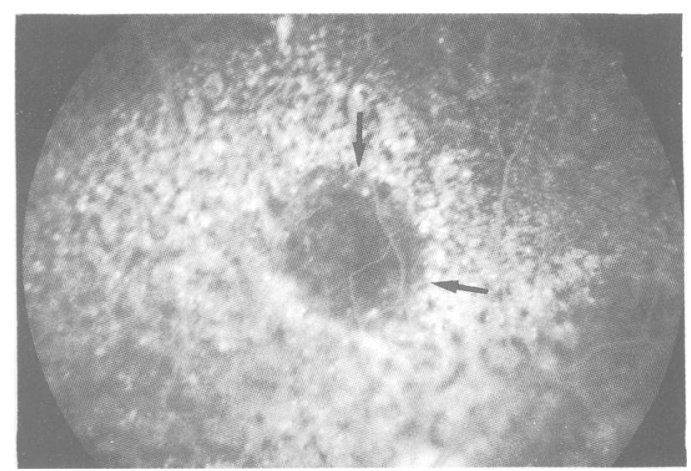

Figure 5 Peripheral fundus (arrows indicate pigmented choroidal lesions). 


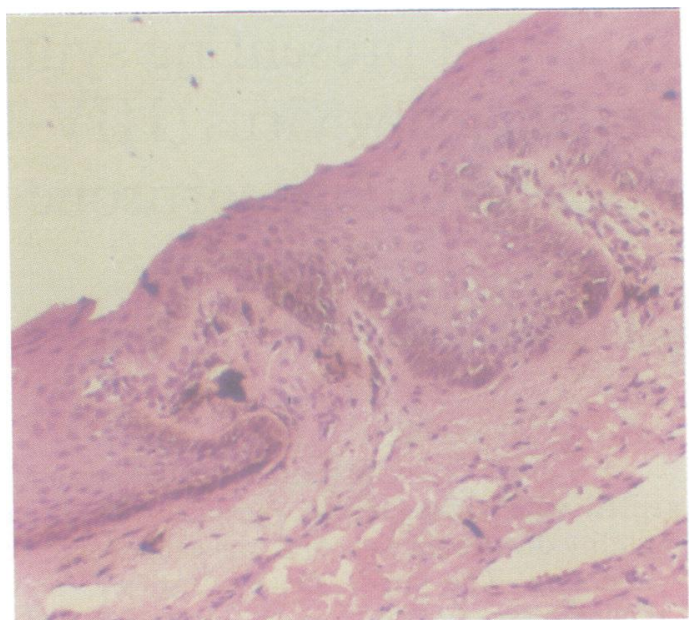

Figure 6 Photomicrograph of lip lesions showing increased numbers of melanocytes in basal layer of epithelium and scattered melanocytes and melanophages in the dermis. (Haematoxylin and eosin, $\times 325$.)

Malignant change in the gastrointestinal polyps occurs in $2-3 \%$ of patients. ${ }^{8}$

Our patient had an occult rectosigmoid adenocarcinoma when he developed pigment oval and penile lesions that had the typical clinical and histopathological appearance of Peutz-Jeghers syndrome. Excision of the colon tumour was followed by rapid development of the typical sequence of signs and symptoms of BDUMP, including visual loss, cataracts, ciliochoroidal detachment, widespread damage to the pigment epithelium and outer retina, appearance of multifocal pigmented uveal tumours, and secondary retinal detachment. Presumably the stimulus for development of multifocal pigmented choroidal lesions and the mucocutaneous lesions was the same. Pigmented choroidal lesions developed over several months and then stabilised in spite of the patient's continuing loss of vision that was associated with progressive pigment epithelial damage and eventually secondary retinal detachment. Simultaneously, the pigmented macules of the mucous membranes and the skin stabilised.

Activation of pre-existing naevus cells by a hormone-producing carcinoma is one of the theories to explain the appearance of multifocal pigmented lesions on a background of selflimited, diffuse, amelanotic melanocytic proliferation throughout the uveal tract of patients with BDUMP. ${ }^{1+}$ There is histopathological evidence that development of the pigmented lesions may be more a product of cancer-induced melanin production and melanocytic necrosis than active cellular proliferation. ${ }^{+}$The same mechanisms may be postulated regarding the appearance of pigmented lesions in the mucous membranes and the skin of our patient. Although there are previous descriptions of BDUMP accompanied by congenital oculocutaneous melanocytosis ${ }^{3}$ and upper respiratory melanosis, our patient is the first to develop extraocular pigmentation concurrently.
This late onset of mucocutaneous pigmentation and the characteristics of this colonic cancer, though not typical of Peutz-Jeghers syndrome, do not exclude it..$^{710}$ Severe visual loss in our patient was associated with extensive pigment epithelial damage prior to development of retinal detachment, a feature noted in other cases of BDUMP. This has been ascribed to a toxic or immune reaction, initiated either by a carcinomauveal melanocytic interaction or directly by substances produced by the carcinoma. ${ }^{+}$This latter mechanism has been suggested as important in the pathogenesis of retinal damage occurring in patients who develop progressive visual loss, nyctalopia, and fundus changes simulating retinitis pigmentosa sine pigmenti in response to a systemic carcinoma, the so-called cancer associated retinopathy (CAR) syndrome. ${ }^{5}$ Our patient had a positive assay for antibodies to retinal cancer-associated antigen. The significance of this is unknown, since a previously reported patient with BDUMP had a negative assay. ${ }^{+}$

No treatment has prevented severe visual loss in patients with BDUMP. Vitrectomy, silicone injection, and panphotocoagulation were successful in partly reattaching our patient's retina, but resulted in only minimal visual improvement. Perhaps this form of therapy should be considered earlier in the course of the disease.

Our patient remained free of evidence of metastasis for 26 months after the onset of his disease. He is the second patient to have survived beyond 24 months after the onset of BDUMP. ${ }^{+}$

Serological examinations for antibodies to cancer-associated Serological examinations for antibodies to cancer-ass

This investigation was supported in part by Public Health This investigation was supported in part by Public Health
Service Research grant EY02549 and core grant EY02180, Service Research grant EY02549 and core grant EY02180,
Department of Health and Human Services, National Institutes of Department of Health and Human Services, National Institutes of Blindness, Inc., New York City

1 Ryll DL, Campbell RJ, Robertson DM, Brubaker SJ. Pseudometastatic lesions of the choroid. Ophthalmology 1980;87: 1181-6.

2 Barr CC, Zimmerman LE, Curtin VT, Font RI. Bilateral diffuse melanocytic uveal tumors associated with systemic malignant neoplasms. Arch Ophthalmol 1982; 100: 249-55.

3 Prause JU, Jensen OA, Eisgart F, Hansen U, Kieffer M. Bilateral diffuse malignant melanoma of the uvea associated Bilateral diffuse malignant melanoma of the uvea associated with large cell carcinoma, giant

4 Gass JDM, Gieser RG, Wilkinson CP, Beahm DE, Pautler SE. Bilateral diffuse uveal melanocytic proliferation in patients with occult systemic carcinoma. Arch Ophthalmol 1990; 108: $527-33$

5 Thirkill CE, Roth AM, Keltner JL. Cancer associated retinopathy. Arch Ophthalmol 1987; 105: 372-5.

6 Peutz JLA. Very remarkable case of familial polyposis of mucous membrane of intestinal tract and nasopharynx accompanied by peculiar pigmentations of skin and mucous membrane. Ned Maandschr Geneeskd 1921;10: 134-6.

7 Jeghers H, McKusick VA, Katz KH. Generalized intestinal polyposis and melanin spots of the oral mucosa, lips and polyposis and melanin spots of the oral mucosa, lips and digits: a syndrome of diagnostic sign

8 Reid JD. Intestinal carcinoma in the Peutz-Jeghers syndrome. ҰAMA $1974 ; 229: 833-4$.

9 Dewan PA, Hope JKA. Peutz-Jeghers syndrome. Aust NZ $\mathcal{F}$ Surg 1985; 55: 613-6.

10 Banse-Kupin LA, Douglass MC. Localization of PeutzJeghers macules to psoriatic plaques. Arch Dermatol 1986; 122: 679-83.

11 Traboulsi EI, Maumenee IH. Periocular pigmentation in the Peutz-Jeghers syndrome. Am $\mathcal{F}$ Ophthalmol 1986; 102: 126 